\title{
Percepción de enfermedad, depresión, ansiedad y control del asma: Una primera aproximación
}

\author{
Isaías Vicente Lugo-González,* Leonardo Reynoso-Erazo, ${ }^{*}$ Margarita Fernández, Vega ${ }^{\S}$ \\ *Programa de Maestría y Doctorado en Psicología. Maestría en Medicina Conductual, UNAM; División de Investigación y Postgrado, \\ Facultad de Estudios Superiores Iztacala, UNAM; ${ }^{\circledR}$ Subdirectora de Enseñanza, INER. \\ Trabajo recibido: 10-IV-2014; aceptado: 26-V-2014
}

\begin{abstract}
RESUMEN. Para favorecer un mejor control del asma se han incluido intervenciones educativas y psicológicas. Uno de los constructos psicológicos más importantes en los últimos años es la percepción de enfermedad, misma que se relaciona con la adherencia al tratamiento, control de la enfermedad e impacto emocional. El presente trabajo tiene como propósito fundamental evaluar la relación entre la percepción de enfermedad, depresión, ansiedad y control de la enfermedad en pacientes con asma. Se realizó un estudio correlacional-transversal en el que participaron 45 pacientes, quienes fueron evaluados sobre la percepción de enfermedad, depresión, ansiedad y control del asma. La mayoría de los pacientes reportó que la enfermedad afectaba severamente su vida y su estado de ánimo. Más del $50 \%$ de los pacientes se encontraban de leve a severamente deprimidos; el $75 \%$ presentaron ansiedad de leve a severa. Se encontraron correlaciones estadísticamente significativas entre depresión y ansiedad; ansiedad y control del asma; impacto de la enfermedad, depresión y ansiedad; percepción de control de la enfermedad y ansiedad; y percepción de impacto emocional de la enfermedad, depresión y ansiedad. Se concluyó que los resultados orientan a marcar la relación entre una forma negativa de percibir la enfermedad con sintomatología de depresión y ansiedad, y control del asma.
\end{abstract}

Palabras clave: Percepción de enfermedad, depresión, ansiedad, control del asma.

ABSTRACT. Educational and psychological interventions are included to promote better asthma control. Recently, one of the most important psychological construct is illness perception, which is related to treatment adherence, disease control and emotional impact. The aim of this study was to evaluate the relationship between illness perception, depression, anxiety and disease control in asthma patients. A correlational cross-sectional study involving 45 patients who were evaluated on illness perception, depression, anxiety and asthma control was performed. Most patients reported that the disease severely affected his (her) life and mood. More than $50 \%$ of the patients were moderate to severely depressed, $75 \%$ had moderate to severe anxiety. Statistically significant correlations between depression and anxiety, anxiety and asthma control, impact of illness, depression and anxiety, perception of disease control and anxiety and perceived emotional impact of illness, depression and anxiety were found. We conclude that the results lead patients to perceive a relationship between disease symptoms, depression, anxiety and asthma control.

Key words: Illness perception, depression, anxiety, asthma control.

\section{INTRODUCCIÓN}

El asma se define como una enfermedad inflamatoria de la vía aérea, crónica, multicausal caracterizada por distintos niveles de severidad. Es una de las enfermedades con más incidencia que genera problemas importantes de morbilidad y mortalidad en todo el mundo. ${ }^{1-4}$

Uno de los principales retos a los que se abocan las guías nacionales e internacionales sobre asma tiene que ver con el esclarecimiento de las vías para el control adecuado de la enfermedad. Se han incluido y desarrollado medicamentos más eficaces, mejoras en la relación médico-paciente, intervenciones educativas que incluyen aspectos psicológicos de la enfermedad para una mejor comprensión de la misma. Así como intervenciones enfocadas a modificar comportamientos asociados a la enfermedad de quien la padece. ${ }^{2-5}$

Los trastornos del estado de ánimo, particularmente depresión y ansiedad, se han investigado de forma rigurosa en los pacientes asmáticos, debido a la alta prevalencia encontrada en esta población y al papel protagónico que parecen tener en el control de la enfermedad. ${ }^{6-11}$

Uno de los aspectos psicológicos más importantes en el que se ha incrementado el número de investigaciones en las enfermedades crónicas, tiene que ver con 
Tabla 1. Cuestionario sobre percepción de enfermedad.

1. ¿Cuáles fueron las causas más importantes por las que según usted enfermó de asma?

2. ¿Cuánto cree que el ASMA afecta su vida?
0
1
23
4
5
6
7
8
9
10

No me afecta

para nada

3. ¿Cuánto control siente tener sobre su enfermedad (ASMA)?

$\begin{array}{llllll}0 & 1 & 2 & 3 & 4 & 5\end{array}$

6

$6 \quad 7$

8

9

Afecta severamente mi vida

Nada

4. ¿Qué tanto apego cree tener a su tratamiento para el ASMA?
0
1
2
3
4

5

6

7

8

9

10

Totalmente

apegado

Nada de apego

5. ¿Qué tan preocupado se encuentra por tener ASMA?

$\begin{array}{cccccccccccc}0 & 1 & 2 & 3 & 4 & 5 & 6 & 7 & 8 & 9 & \begin{array}{c}10 \\ \text { Estoy muy } \\ \text { preocupado }\end{array} \\ \text { preocupado } & & & & & & & & & & \end{array}$

6. ¿Cuánto le afecta emocionalmente tener ASMA? (Es decir, ¿lo hace sentirse enojado, molesto, asustado, preocupado o deprimido?)

0

12

3

4

$5 \quad 6$

7

8

9

10

No me afecta

Estoy muy afectado

la percepción de enfermedad, constructo desarrollado a partir del modelo de sentido común y de autorregulación de la enfermedad (MSC). De acuerdo con este modelo, cada paciente organiza, explica y describe información perceptiva (síntomas) o conceptual (diagnóstico) de manera variable y dependiendo de experiencias y por medio de la retroalimentación constante. El resultado de esta organización es la elaboración de una representación cognitiva/emocional del problema (síntomas o enfermedad), seguida de una puesta en marcha de acciones (búsqueda o no de atención médica, uso o no de medicamentos) y finalizada por la evaluación de las acciones llevadas a cabo..$^{12,13}$

La percepción de enfermedad, luego entonces, tiene que ver con una representación cognitiva organizada, o conjunto de creencias en relación y sobre la enfermedad, sus síntomas y el tratamiento. ${ }^{14}$ Esta percepción se construye con base en la información previa que se tenga de la enfermedad, información proporcionada por el equipo médico, otros pacientes o de los medios de comunicación, así como con diversas experiencias significativas durante el proceso de enfermedad o previas a ella. ${ }^{15}$

Se han identificado dos componentes que conforman la percepción de enfermedad: 1) componente emocional (depresión, ansiedad, enojo, miedo, como resultado de padecer síntomas de la enfermedad o llevar a cabo un tratamiento específico) y 2) componente cognitivo (creencias sobre la identidad, causas, temporalidad, consecuencias y control/cura de la enfermedad). ${ }^{15,16}$ Dicho de otra forma, el comportamiento de una persona ante la enfermedad o sus síntomas (adaptación, control y manejo) estará en función de las creencias que tenga sobre los mismos, y las reacciones emocionales derivadas de la sintomatología y significado del padecimiento..$^{14-17}$

La percepción de enfermedad se ha estudiado en diversos padecimientos, por citar algunos ejemplos: diabetes y creencias sobre el uso de medicamentos, ${ }^{18}$ enfermedad pulmonar obstructiva crónica, ${ }^{19,20}$ osteoartritis, ${ }^{21}$ enfermedad renal crónica ${ }^{22}$ y asma; de manera específica en este último padecimiento, se ha observado que existe relación entre el tipo de percepción de enfermedad y diversas conductas de automanejo, adherencia al tratamiento, afrontamiento, adaptación a la enfermedad, así como los resultados en las variables de evaluación médica. 15,17,23,24

Objetivo. Evaluar la relación entre la percepción de enfermedad, depresión, ansiedad y control de la enfermedad en pacientes con asma. 


\section{MATERIAL Y MÉTODOS}

Participantes: 45 pacientes con asma atendidos en la Clínica de Asma y del Servicio Clínico No. 5 del Instituto Nacional de Enfermedades Respiratorias Ismael Cosío Villegas (INER) de la Ciudad de México. Muestreo: Se utilizó un muestreo no probabilístico de sujetos voluntarios. ${ }^{25}$ Diseño: Fue realizado a través de un estudio correlacional-trasversal. Instrumentos: Entrevista para pacientes con asma (diseñada ad hoc como complemento de otra investigación) que incluyó preguntas sobre datos sociodemográficos, clínicos de la enfermedad, seis preguntas sobre percepción de enfermedad basadas en el Cuestionario Breve de Percepción de Enfermedad versión en español (B-IPQ). ${ }^{16}$ La pregunta 1, correspondiente a las causas de la enfermedad fue abierta y en ella se pidió a los pacientes que describieran, según ellos, las causas más importantes por las que enfermaron de asma; y la instrucción del resto de las preguntas fue que respondieran en una escala numérica del 0-10 (tabla 1).

Inventario de depresión de Beck (IDB): Cuestionario de 21 reactivos para evaluar la severidad de la sintomatología depresiva, por cada ítem la persona puede seleccionar entre cuatro posibles alternativas que mejor refleje su situación durante la última semana; estas alternativas van de no me siento a siempre me siento o de nada a muy intenso. La puntuación total se calculó mediante la suma de todos los ítems y osciló en un rango de 0 a 63 puntos: depresión mínima (0-9), leve (10-16), moderada (17-29) y severa (30-63). ${ }^{26}$

Inventario de ansiedad de Beck (IAB): Cuestionario de 21 reactivos para evaluar la severidad de la sintomatología ansiosa. Cuenta con cuatro opciones de respuesta, en la que 0 significa poco o nada y 3 severamente. La puntuación total se calculó mediante la suma de todos los ítems y osciló en un rango de 0 a 63 puntos: ansiedad mínima (0-5), leve (6-15), moderada (16-30) y severa (31-63). ${ }^{27}$

Test del control del asma, ACT, (del inglés asthma control test): Cuestionario heteroaplicable, ${ }^{28}$ para evaluar el control del asma durante cuatro semanas previas.
Consta de cinco preguntas con una escala tipo Likert de cinco puntos. La puntuación máxima es 25 , misma que corresponde a asma controlada; de 20 a 24 puntos corresponde a asma parcialmente controlada; y una puntuación igual o menor a 19 corresponde a asma no controlada. ${ }^{29}$

\section{Procedimiento}

Consulta externa: La recolección de los datos fue a través de la Clínica de Asma del Instituto Nacional de Enfermedades Respiratorias Ismael Cosío Villegas (INER), México, se contó con una lista de los pacientes que pasarían a consulta con el neumólogo. Se llamó a cada paciente presentándose con él y se explicó la realización de la investigación y cuál era el objetivo.

Hospitalización: Para la recolección de los datos en el Servicio Clínico 5, INER, se revisaron los kardex y se acudió con los pacientes que tuvieran diagnóstico de asma y hubieran sido hospitalizados por crisis de asma. Se les explicó la realización de la investigación y su objetivo.

Si los pacientes de la consulta externa y hospitalización aceptaban participar en la investigación, entonces se les pedía que leyeran y firmaran el consentimiento bajo información. Así se procedió a la aplicación de los cuestionarios.

\section{RESULTADOS}

Se realizaron análisis descriptivos y de correlación con el programa estadístico SPSS v20 para Windows.

\section{Características sociodemográficas y clínicas}

En la tabla 2 se muestran las características sociodemográficas y clínicas de los pacientes que participaron en la investigación. El número mayor de participación fueron mujeres, la edad mínima fue de 18 años, la mayoría eran casados y divorciados. El nivel educativo se distribuyó de forma homogénea entre primaria y licenciatura; y la media en tiempo de evolución de la enfermedad fue de aproximadamente 10 años.

Tabla 2. Características demográficas y clínicas de los pacientes.

\begin{tabular}{|c|c|c|c|c|c|c|c|c|c|c|}
\hline \multicolumn{3}{|c|}{ Sexo (\%) } & \multicolumn{2}{|c|}{ Edad años } & \multicolumn{3}{|c|}{ Estado civil } & \multicolumn{2}{|c|}{ Escolaridad (\%) } & Tiempo asma (\%) \\
\hline Femenino & & (71.1) & Mediana & 36 & Casado & 19 & $(42.2)$ & Primaria & $11(24.4)$ & Media 119.67 (meses) \\
\hline \multirow[t]{5}{*}{ Masculino } & 13 & (28.9) & Mínimo & 18 & Soltero & 15 & (33.3) & Secundaria & $11(24.4)$ & Mínimo 1 \\
\hline & & & Máximo & 77 & Divorciado & 5 & (11.1) & Preparatoria & $9(20)$ & Máximo 360 \\
\hline & & & & & Unión libre & 5 & (11.1) & Licenciatura & $9(20)$ & \\
\hline & & & & & Viudo & 1 & $(2.2)$ & Analfabeta & $3 \quad(6.7)$ & \\
\hline & & & & & & & & Postgrado & $(4.4)$ & \\
\hline
\end{tabular}


Tabla 3. Causas asociadas a enfermar de asma.

\begin{tabular}{|c|c|c|c|}
\hline Pregunta & Área & Respuestas & $\%$ \\
\hline 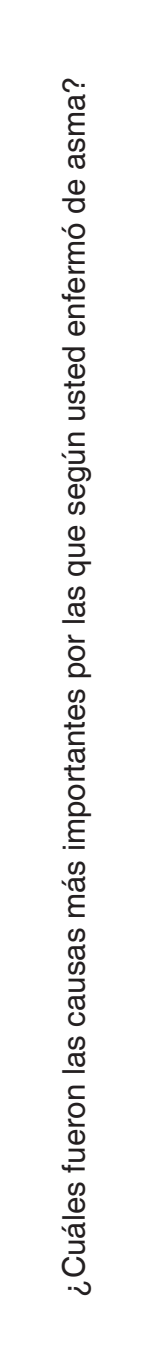 & $\begin{array}{l}\text { Emocionales } \\
\text { Enfermedades respiratorias } \\
\text { Clima } \\
\text { Animales } \\
\text { Alergias } \\
\text { Trabajo } \\
\text { Ambiente } \\
\text { Herencia } \\
\text { Sustancias } \\
\text { Plantas } \\
\text { Nacimiento } \\
\text { Conocimientos }\end{array}$ & $\begin{array}{l}\text { Descuido } \\
\text { No acudir al médico a tiempo } \\
\text { No usar los medicamentos } \\
\text { Mojarse } \\
\text { No taparse } \\
\text { Bañarse y lavar ropa temprano } \\
\text { Fumar } \\
\text { Usar drogas } \\
\text { Auto-medicarse } \\
\text { Cocinar con leña } \\
\text { Tomar Desenfriol } \\
\text { Comer chocolate } \\
\text { Por problemas familiares } \\
\text { Tristeza } \\
\text { El carácter } \\
\text { Depresión } \\
\text { Los nervios } \\
\text { Inestabilidad emocional } \\
\text { La soledad } \\
\text { Enfermarse frecuentemente de gripe } \\
\text { Gripes mal cuidadas } \\
\text { Exposición al frío } \\
\text { Convivir con animales y mascotas } \\
\text { Antecedentes de alergias } \\
\text { Exposición laboral a pintura, campo y madera } \\
\text { Exposición al medio ambiente (contaminación) } \\
\text { Antecedentes hereditarios } \\
\text { Exposición al cloro } \\
\text { Exposición a plantas } \\
\text { De nacimiento } \\
\text { Falta de conocimientos }\end{array}$ & $\begin{array}{c}18 \\
17.6 \\
11 \\
11 \\
8.8 \\
6.6 \\
4.4 \\
4.4 \\
2.2 \\
2.2\end{array}$ \\
\hline
\end{tabular}

En lo que respecta a la ocupación de los pacientes, el $31 \%$ se dedicaban al hogar, el $17.8 \%$ eran empleadas domésticas y de igual manera profesionistas, el $11 \%$ estudiantes, el $9 \%$ empleados y otro $13 \%$ se dedicaban a otra actividad como el comercio o diversos oficios. El 95\% vivían con algún familiar y el resto vivían solos. El $77.8 \%$ de los pacientes fueron captados en consulta externa y el $22.2 \%$ en hospitalización.

\section{Percepción de enfermedad}

En la tabla 3 se describe la categorización de las respuestas a la pregunta 1 sobre las causas de enfermar de asma; y en la tabla 4, la distribución de las respuestas entre 0-10 en porcentaje para el resto del cuestionario.

Las causas asociadas a enfermar de asma están agrupadas a diferentes áreas; éstas fueron conformadas por la categorización de las respuestas de los pacientes. Por ejemplo, el área denominada conductual está conformada por respuestas asociadas a llevar a cabo una conducta (fumar, automedicarse, mojarse, etc.), omisión de una conducta (no acudir al médico a tiempo, no usar medicamentos) o un conjunto de conductas (bañarse y lavar temprano, cocinar con leña). En lo que respecta al área emocional, está conformada por respuestas relacionadas con estados emocionales y situaciones que incluyen consecuencias emocionales negativas. 
Tabla 4. Distribución de las respuestas para el cuestionario.

\begin{tabular}{|c|c|c|c|c|c|}
\hline Pregunta & 0123 & 456 & 78910 & $x$ & DE \\
\hline $\begin{array}{c}\text { ¿Cuánto cree que el ASMA afecta } \\
\text { su vida? }\end{array}$ & $\begin{array}{c}\text { No me afecta nada } \\
26.7 \%\end{array}$ & $31.1 \%$ & $\begin{array}{l}\text { Afecta severamente mi vida } \\
\qquad \mathbf{4 2 . 1 \%}\end{array}$ & 5.16 & 3.04 \\
\hline $\begin{array}{c}\text { ¿Cuánto control siente tener sobre } \\
\text { su enfermedad? }\end{array}$ & $\begin{array}{c}\text { Nada de control } \\
6.6 \%\end{array}$ & $31.3 \%$ & $\begin{array}{l}\text { Totalmente bajo control } \\
62.2 \%\end{array}$ & 7.07 & 2.58 \\
\hline $\begin{array}{l}\text { ¿Qué tanto apego cree tener a su } \\
\text { tratamiento para el ASMA? }\end{array}$ & $\begin{array}{c}\text { Nada de apego } \\
4.4 \%\end{array}$ & $22.2 \%$ & $\begin{array}{c}\text { Totalmente apegado } \\
\mathbf{7 1 . 1 \%}\end{array}$ & 7.11 & 2.00 \\
\hline $\begin{array}{c}\text { ¿Qué tan preocupado se encuentra } \\
\text { por tener ASMA? }\end{array}$ & $\begin{array}{c}\text { No estoy preocupado } \\
\mathbf{3 1 \%}\end{array}$ & $15.5 \%$ & $\begin{array}{l}\text { Estoy muy preocupado } \\
53.3 \%\end{array}$ & 5.93 & 3.51 \\
\hline $\begin{array}{c}\text { ¿Cuánto le afecta emocionalmente } \\
\text { tener ASMA? }\end{array}$ & $\begin{array}{c}\text { No me afecta nada } \\
\mathbf{2 8 . 8 \%}\end{array}$ & $28.8 \%$ & $\begin{array}{c}\text { Estoy muy afectado } \\
42.2\end{array}$ & 5.13 & 3.53 \\
\hline
\end{tabular}

X = Media; DE = Desviación estándar.

Según se observa, los porcentajes más altos asociados a las principales causas de enfermedad tienen que ver con acciones que llevaron a cabo, acciones que no llevaron a cabo y estados emocionales, que los pacientes evalúan como determinantes de padecer asma. La mayoría de los pacientes reportaron que la enfermedad llega a afectar severamente su vida, estar muy preocupados por la enfermedad y encontrarse afectados emocionalmente. Pero de la misma forma, la mayoría reportó tener totalmente bajo control su enfermedad y estar totalmente apegados al tratamiento.

\section{Depresión, ansiedad y control del asma}

En lo que respecta a las puntuaciones en depresión, ansiedad y control del asma, en la tabla 5 se muestran las medidas de tendencia central para las puntuaciones que obtuvieron los pacientes en los instrumentos, y porcentaje de pacientes distribuidos en los niveles de severidad (depresión y ansiedad) y control del asma. Más del $50 \%$ de los pacientes se encontraban de leve a severamente deprimidos, el $75 \%$ presentó ansiedad de leve a severa. Asimismo, el $42.2 \%$ de los pacientes que presentaron depresión mínima (>10 puntos) también presentaron un nivel de ansiedad mínima (a partir de 10 puntos).

En cuanto a los resultados de las correlaciones entre percepción de enfermedad, depresión, ansiedad y control del asma, se encontró correlación positiva estadísticamente significativa entre depresión y ansiedad $(r=.709, p=.000)$, una correlación negativa estadísticamente significativa entre ansiedad y control del asma $(r=-.422, p=.004)$, no hubo correlación entre depresión y control del asma $(r=-118, p=.439)$.

Por otro lado, se encontró correlación estadísticamente significativa entre la percepción de impacto
Tabla 5. Puntuaciones obtenidas en el IDB, IAB, ACT.

\begin{tabular}{|c|c|c|c|c|}
\hline & IDB & $\mathrm{IAB}$ & & ACT \\
\hline $\mathrm{X}$ & 12.89 & 14.42 & $\mathrm{X}$ & 16.6 \\
\hline \multirow[t]{2}{*}{ DE } & 11.260 & 10.616 & $\mathrm{DE}$ & 4.343 \\
\hline & \multicolumn{2}{|c|}{ Severidad } & \multicolumn{2}{|c|}{ Control del asma } \\
\hline Mínima & $46.6 \%$ & $24.3 \%$ & No controlada & $84.4 \%$ \\
\hline Leve & $24.3 \%$ & $35.3 \%$ & Parcialmente controlada & $13.3 \%$ \\
\hline Moderada & $15.4 \%$ & $28.6 \%$ & Controlada & $2.2 \%$ \\
\hline Severa & $13.2 \%$ & $11 \%$ & & \\
\hline
\end{tabular}

$\mathrm{X}=$ Media; $\mathrm{DE}=$ Desviación estándar; IDB = Inventario de Depresión de Beck; IAB = Inventario de Ansiedad de Beck; $A C T$ = Asthma control test (por sus siglas en inglés).

del asma en la vida del paciente, depresión ( $r=.486$, $\mathrm{p}=.001)$ y ansiedad $(\mathrm{r}=.536, \mathrm{p}=.000)$, correlación negativa entre la percepción de control de la enfermedad y ansiedad $(r=-.295, p=.049)$, correlación negativa entre la percepción de apego al tratamiento y depresión y $(r=-.317, p=.034)$, correlación entre preocupación por la enfermedad, depresión ( $r=.407, p=.005)$ y ansiedad $(r=.365, p=.014)$ e impacto emocional de la enfermedad y depresión $(r=.501, p=.000)$ y ansiedad $(r=.564$, $p=.000$ ).

\section{DISCUSIÓN}

El propósito de este trabajo fue evaluar la existencia de relación entre la percepción de enfermedad, depresión, ansiedad y control del asma en un grupo de pacientes del INER. Cumpliendo el objetivo antes expuesto, en esta primera aproximación, los resultados obtenidos se orientan a la relación entre la forma de percibir algunos elementos de la enfermedad (asma), 
como la valoración del impacto negativo en la vida del paciente, las repercusiones emocionales de la enfermedad, la valoración del control de la enfermedad y el apego al tratamiento, con la sintomatología de depresión y ansiedad y asociación negativa entre ansiedad y control del asma.

Algunos resultados similares se muestran en la investigación realizada por DiMarco et al.,$^{30}$ en la que evaluaron la relación entre ansiedad, depresión y control del asma, encontrando relaciones importantes entre ansiedad, depresión y descontrol en la enfermedad.

\section{Creencias sobre la enfermedad}

Otros resultados que llaman la atención, es la variabilidad de causas referidas por los pacientes como asociadas a padecer asma. Algunas tiene que ver con una lógica hacia enfermedades respiratorias y alergias, conductas relacionadas a padecimientos respiratorios y el tipo de actividad laboral y de convivencia asociadas a repercusiones en el sistema respiratorio (más del 60\% de los pacientes evaluados las refirieron como una de las causas de su enfermedad). Otras, con acciones aparentemente incompatibles a padecimientos respiratorios como el consumo de chocolate (sólo en pacientes alérgicos al chocolate), medicamentos, la hora de la higiene personal y labores del hogar. Y también las asociadas a las causas «temperamentales», «psicológicas» o «emocionales», éstas últimas, en particular, son hasta cierto grado de dominio popular, ya que el asma se ha asociado con causas "psicosomáticas o como una enfermedad dominada por la mente", y en ocasiones se siguen refiriendo como causa determinante de padecer este tipo de enfermedad crónica. ${ }^{31}$

Interesante es también que los pacientes que por un lado valoran como causa de enfermedad los problemas respiratorios, asimismo, consideran que la inestabilidad emocional y algunos tipos de conductas específicas son causas asociadas.

Al conocer estas creencias sobre la enfermedad se pone de manifiesto las atribuciones del origen de la misma, historia de la enfermedad, información y conocimientos disponibles, necesidades educativas, así como probables conductas de afrontamiento y automanejo. Ejemplo de lo anterior es la investigación realizada por Halm et al., ${ }^{23}$ en la que evaluaron las creencias de asma episódica o crónica de 198 adultos con asma, concluyendo que más del $50 \%$ refirió sólo tener asma cuando tenían síntomas, $20 \%$ refirió que no padecerían asma para siempre y $50 \%$ creían que su médico los curaría de la enfermedad. Asimismo, el $50 \%$ creían que los corticoesteroides inhalados no eran necesarios cuando no se tenían síntomas, no acostum- braban visitar a su médico si estaban asintomáticos, ni usaban el flujómetro.

Por su parte, Mueller et al., ${ }^{32}$ evaluaron la percepción de salud y enfermedad de 97 jóvenes con diversos padecimientos, entre los que se incluía asma, concluyendo que las creencias y actitudes positivas y flexibles hacia la enfermedad, favorecen la adaptación a la misma. Además, aquellos jóvenes que creían que su estado de salud no era algo modificable asociaban su bienestar con la herencia, se involucraban menos en conductas saludables como realizar actividad física y eran más propensos a considerarse menos saludables que los jóvenes que consideraban su estado de salud como modificable.

\section{Control de la enfermedad y percepción de enfermedad}

De acuerdo con Leventhal et al., las características sociodemográficas como la edad, acceso a servicios de salud y condiciones económicas, no determinan del todo el manejo de la enfermedad. Sin embargo, muchas veces se hace referencia a este tipo de factores como responsables de los problemas en el automanejo y control de la enfermedad. Es necesario mencionar que este tipo de condiciones se asocian con factores en los que en ocasiones, resulta imposible realizar modificaciones. En este sentido, en los últimos años se ha optado por el acercamiento a factores más de tipo proximal, como son los factores individuales y en los que se pueden generar cambios de manera más accesible, en este caso la percepción de enfermedad. ${ }^{15}$

Resultados encontrados en esta investigación sostienen que la mayoría de los pacientes evaluados perciben tener control sobre su enfermedad; asimismo, también refieren estar totalmente apegados a su tratamiento para el asma. Sin embargo, esto genera consideraciones importantes: 1) si perciben tener control de la enfermedad y estar totalmente apegados al tratamiento, seguramente estarían controlados de acuerdo con el $\mathrm{ACT},{ }^{27}$ y los resultados en este sentido muestran lo contrario, y 2) al estar controlados y apegados al tratamiento, experimentarían pocos o ningún síntoma de asma, favoreciendo una buena calidad de vida y minimizando repercusiones emocionales, comúnmente observadas en pacientes descontrolados. ${ }^{7,10,11,30}$ Del mismo modo, los resultados sobre depresión, ansiedad y la percepción de impacto emocional de la enfermedad, también muestran lo contrario.

Lo anterior es ejemplo de que el modo de percibir diferentes aspectos de la enfermedad como síntomas, repercusiones emocionales o el tratamiento, no son 
del todo funcionales para llevar un adecuado control del padecimiento. De aquí, la necesidad de realizar intervenciones conductuales en educación para la salud y percepción de la enfermedad.

En el caso del asma, en el MSC ${ }^{12}$ se proponen dos maneras de favorecer cambios en la percepción de la enfermedad: 1) el uso de estrategias abstracta/ cognitivas (top-down) orientadas a favorecer una percepción sobre el asma como enfermedad presente aún con la ausencia de síntomas y controlable con la implementación de conductas concretas. La necesidad de este cambio cognitivo es debido a que algunos pacientes pueden creer no necesitar el medicamento al no presentar síntomas, ${ }^{23}$ creencia incompatible con el uso continuo de medicamentos en el tratamiento para el asma, y 2) el uso de conductas/concretas (bottom-up, uso de medicamentos, flujómetro, registro de síntomas, control ambiental, etc.); y así, favorecer la detección de los efectos de este tipo de conductas, mismos que modificarán su experiencia con la enfermedad. ${ }^{33}$

Los resultados obtenidos sugieren información importante sobre: 1) la atribución del origen y causas de la enfermedad y la indudable necesidad de la educación en asma; 2) el probable valor predictivo de la valoración y percepción de la enfermedad en relación con el impacto emocional del padecimiento y apego al tratamiento médico, y 3) la necesidad de incluir y valorar la percepción de enfermedad en el tratamiento cotidiano de los pacientes, y para el desarrollo de intervenciones en este sentido.

\section{Limitaciones del estudio}

Encontramos dos limitaciones importantes en el estudio, a saber: 1) el número de pacientes evaluados fue limitado, tomando en cuenta la gran cantidad de pacientes atendidos en el INER, y 2) incluir un número limitado de pacientes hospitalizados que pudieran sesgar las variables evaluadas. Para futuras investigaciones se pretende aumentar el número de pacientes que participen, y realizar comparaciones entre pacientes de consulta externa, hospitalizados y urgencias y determinar si existe diferencia en la percepción de enfermedad. También sería importante evaluar si la percepción de enfermedad en los pacientes con asma guarda relación no sólo con aspectos psicológicos, sino también de calidad de vida, visitas a urgencias, uso de servicios médicos y manejo de asma, conductas de autocuidado y marcadores biológicos como el conteo eosinofílico y de cortisol. Correlaciones encontradas en el trabajo de Walker y Chen. ${ }^{24}$

\section{REFERENCIAS}

1. Ortega MJA, Fernández VM. Diagnóstico de asma. Neumol Cir Torax 2009;68(Supl 2):S116-S122.

2. Sociedad Mexicana de Neumología y Cirugía de Tórax. Consenso mexicano de asma. Neumol Cir Torax 2005;64(Supl 1):S7-S44.

3. National Heart, Lung, and Blood Institute. National asthma education and prevention program. Expert panel report 3: guidelines for the diagnosis and management of asthma 2007. Access date: 2012 April 15. Available from: www.nhlbi.org

4. Global Initiative for Asthma. Global strategy for asthma management and prevention 2011. Access date: 2012 April 15. Available from: www.ginasthma.org

5. Fernández VM, Amaya BL, Martínez PJ. Educación en asma. Neumol Cir Torax 2009;68(Supl 2):S176-S185.

6. Lehrer PM. Emotionally triggered asthma: a review of research literature and some hypotheses for selfregulation therapies. Appl Psychophysiol Biofeedback 1998;23(1):13-41.

7. Lehrer P, Feldman J, Giardino N, Song HS, Schmaling K. Psychological aspects of asthma. J Consult Clin Psychol 2002;70(3):691-711.

8. Goodwin RD, Fergusson DM, Horwood LJ. Asthma and depressive and anxiety disorders among young persons in the community. Psychol Med 2004;34(8):1465-1474.

9. Opolski M, Wilson I. Asthma and depression: a pragmatic review of the literature and recommendations for future research. Clin Pract Epidemiol Ment Health 2005;1(18):1-7.

10. Yorke J, Fleming SL, Shuldham C. Psychological interventions for adults with asthma: a systematic review. Respir Med 2007;101(1):1-14.

11. Van Lieshout RJ, Macqueen G. Psychological factors in asthma. Allergy Asthma Clin Immunol 2008;4(1):12-28.

12. Leventhal $\mathrm{H}$, Brissette I, Leventhal $\mathrm{E}$. The common sense model of self-regulation of health and illness. In: Cameron LD, Leventhal $\mathrm{H}$, editors. The self-regulation of health and illness behavior. Londres: Taylor \& Francis Books; 2003.p.42-65.

13. Beléndez M, Bermejo RM, García MD. Estructura factorial de la versión española del Revised IIIness Perception Questionnaire en una muestra de sujetos con hipertensión. Psicothema 2005;17(2):318-324.

14. Petrie KJ, Jago LA, Devcich DA. The role of illness perceptions in patients with medical conditions. Curr Opin Psychiatry 2007;20(2):163-167.

15. Kaptein AA, Klok T, Moss-Morris R, Brand PL. Illness perceptions: impact on self-management and control in asthma. Curr Opin Allergy Clin Immunol 2010;10(3):194-199.

16. Broadbent E, Petrie KJ, Main J, Weinman J. The brief illness perception questionnaire. J Psychosom Res 2006;60(6):631-637.

17. Kaptein AA, Hughes BM, Scharloo M, et al. Illness perceptions about asthma are determinants of outcome. J Asthma 2008;45(6):459-464.

18. Mann DM, Ponieman D, Leventhal H, Halm EA. Predictors of adherence to diabetes medications: the 
role of disease and medication beliefs. J Behav Med 2009;32(3):278-284.

19. Scharloo M, Kaptein AA, Schlösser M, et al. Illness perceptions and quality of life in patients with chronic obstruvtive pulmonary disease. J Asthma 2007;44(7):575-581.

20. Kaptein AA, Scharloo M, Fischer MJ, et al. Illness perceptions and COPD: an emerging field for COPD patient management. J Asthma 2008;45(8):625-629.

21. Kaptein AA, Bijsterbosch J, Scharloo M, Hampson SE, Kroon HM, Kloppenburg M. Using the common sense model of illness perceptions to examine osteoarthritis change: a 6-year longitudinal study. Health Psychol 2010;29(1):56-64.

22. Lin CC, Chen MC, Hsieh HF, Chang SC. Illness representations and coping processes of Taiwanese patients with early-stage chronic kidney disease. J Nurs Res 2013;21(2):120-128.

23. Halm EA, Mora P, Leventhal H. No symptoms, no asthma: the acute episodic disease belief is associated with poor self-management among inner-city adults with persistent asthma. Chest 2006;129(3):573-580.

24. Walker HA, Chen E. The impact of family asthma management on biology: a longitudinal investigation of youth with asthma. J Behav Med 2010;33(4):326-334.

25. Hernández R, Fernández C, Baptista P. Metodología de la investigación. 4ta. ed. México: Mc Graw-Hill; 2008.

26. Jurado S, Villegas ME, Méndez L, Rodríguez F, Loperena $\mathrm{V}$, Varela $\mathrm{R}$. La estandarización del Inventario de Depresión de Beck para los residentes de la ciudad de México. Salud Mental 1998;21(3):26-31.

27. Robles R, Verela, R, Jurado S, Páez F. Versión mexicana del Inventario de Ansiedad de Beck: propiedades psicométricas. Rev Mex Anal Conduct 2001;34:313-331.
28. Nathan RA, Sorkness CA, Kosinski M, et al. Development of the asthma control test: a survey for assessing asthma control. J Allergy Clin Immunol 2004;1138(1):59-65.

29. El Hasnaoui A, Martin J, Salhi H, Doble A. Validation of the Asthma Control Test questionnaire in a North African population. Respir Med 2009;103 Suppl 2:S30-S37.

30. Di Marco F, Verga M, Santus P, et al. Close correlation between anxiety, depression, and asthma control. Respir Med 2010;104(1):22-28.

31. Guillen-Díaz C, Lugo I. El tratamiento psicológico del asma en el campo de la medicina conductual. En: Reynoso-Erazo L, Becerra AL, editores. Teoría y práctica de la medicina conductual. 2014. En prensa.

32. Mueller C, Williams R, Dweck C. Children's theories of health predict their responses to disease. J Surg Res 2010;158(2):368-372.

33. McAndrew LM, Musumeci-Szabó TJ, Mora PA, et al. Using the common sense model to design interventions for the prevention and management of chronic illness threats: from description to process. Br J Health Psychol 2008;13(Pt 2):195-204.

\author{
$\triangle$ Correspondencia: \\ Mtro. Isaías Vicente Lugo González \\ Calle Cedros. Andador 7C. Lote 7. Manzana 7. \\ Condominio C. Colonia Polígono 5. 55176. Ecatepec \\ de Morelos. Estado de México. \\ Correo electrónico: isaiasvice@hotmail.com
}

El autor declara no tener conflicto de intereses. 